\title{
Presentación \\ La rebelión de los objetos y de los significados
}

El presente número del Boletín es, desde varios aspectos, único. Por primera vez reunimos un conjunto tan extenso como variado de artículos y estudios que abarcan, además, una zona geográfica y un período de tiempo igualmente vastos. Es un número especial también, porque aparece en el complejo momento que atraviesa nuestro país. En un escenario de confrontaciones y emergencia de nuevas identidades, significados y visiones de mundo, las contribuciones que llenan las siguientes páginas nos muestran, de cierta manera, latísimos procesos de resistencia de distintos grupos sociales. Dichos procesos se plasmaron, de modo semejante a los de hoy, en los distintos objetos a través de los cuales arqueólogos, antropólogos y etnohistoriadores buscan reconstruir nuestro pasado. Hoy nuestras ciudades han cambiado, el sentido ha mutado, la iconografía que nos representa acoge nuevos colores y formas. Todo objeto se transforma en una página sobre la cual expresar intensamente todo lo que nos sucede. Por haber sido parte de este momento, agradecemos a los autores y autoras por su paciencia y a los evaluadores por no abandonarnos en esta labor.

El primer grupo está conformado por tres artículos cuyos temas de investigación se ubican geográficamente en el actual Perú. El primero de ellos, de Denis Correa-Trigoso y Fabián Soberón, presenta los avances en las excavaciones realizadas en la costa norte del Perú, en el Complejo Arqueológico de Chan Chan, específicamente en los patios norte del conjunto amurallado de Chol An. Los autores buscaron determinar la función de estos espacios arquitectónicos con el propósito de establecer una secuencia de construcción y ocupación de este sector. Sus conclusiones les permiten establecer cuatro momentos constructivos: los dos primeros relacionados con la edificación inicial del conjunto amurallado, el tercero vinculado con el pleno desarrollo de las actividades culturales y, el cuarto, caracterizado por la reocupación posterior al abandono de Chan Chan.

Un poco más hacia el sur, abordando el estudio arquitectónico de la estética Chancay, Miguel Guzmán y Jorge Alvino identifican y explican dicha estética a partir del estudio de sus componentes formales, llegando a plantear una tipología particular. La propuesta de los autores tiene como eje central los principios del diseño de esta cultura y sus expresiones decorativas, lo que les permite esbozar un análisis formal, funcional y simbólico. Su investigación plantea la existencia no solo de una estética arquitectural chancay, sino además, de estrechos lazos entre ella y las sociedades andinas con las que coexistió. Así, con respecto a los principios del diseño, algunos de los más relevantes serían la simetría yanantin, alternancia y ritmo, profundidad y repetición. 
Cerrando este primer grupo de artículos, José Luis Martínez aborda el problema de la rebelión de Manco Inca desde Vilcabamba, ocurrida en pleno siglo XVI, a través de fuentes de distinta naturaleza: visuales y orales. A lo largo de su investigación, el autor establece dos momentos en la construcción de la narrativa andina sobre el Inca, apuntando a la autonomía discursiva de cada uno de esos soportes. Martínez plantea la necesidad de la construcción de una metodología de trabajo a partir de las fuentes andinas que, si bien pudieron ser impactadas por las formas comunicacionales europeas, mantuvieron sus principios, lenguajes y categorías de significación propias, además de apelar a comunidades de sentido que se iban adaptando a los vertiginosos cambios coloniales.

Pasando al otro lado de la cordillera de los Andes, y encabezando el segundo grupo de artículos, esta vez enfocados en el estudio de materiales argentinos, María Gabriela Ortiz nos traslada al Período Formativo jujeño. Lo hace a través de las pipas de estilo "San Francisco", conjunto de objetos arqueológicos que presentan particulares diseños y manufactura, distinguiéndolas de otras del Noroeste de Argentina. Dada la escasa información contextual de estas pipas, la autora propone un acercamiento desde la mirada etnográfica de las prácticas shamánicas y las considera como parte de una tecnología ritual, es decir, objetos sacralizados que tienen una vida antes de transformarse en basura ritual. Por otra parte, Ortiz se refiere también a la iconografía de las pipas, que presentan personajes con una corporalidad de extraordinaria regularidad representacional, lo que le permite suponer que estos habrían sido "seres auxiliares" que apoyaban al shamán en sus labores rituales.

Flavia Ottalagano, María Reinoso y Eleonora Freire abordan el estudio del estilo GoyaMalabrigo, presente en la cuenca del Paraná Medio e Inferior y también en algunos sectores de los ríos Uruguay y de La Plata. Las autoras se concentran en la confección de las asas figurativas, cuyo motivo más recurrente son las "cabezas de loro". Gracias al pionero uso combinado de RS Y XRD, las autoras identifican la composición de las mezclas pigmentarias presentes en la cerámica monocroma, destacando el uso de hematita, maghemita, hidroxiapatita, illita, montmorillonita, anhidrita y dióxido de titanio. Sus hallazgos constituyen un aporte relevante para el conocimiento tecnológico cerámico de los grupos humanos que habitaron en los sectores mencionados.

Analizando alrededor de 800 grabados incisos lineales, hoyuelos y tacitas presentes en 25 rocas al descubierto, María José Ołs, Andrés Rocha, Lourdes Iniesta y Pablo Cahiza abordan el estudio del arte rupestre, posiblemente de función ritual, en el sitio La Pampa, piedemonte del Río de Las Tunas, Mendoza, en el marco de la Arqueología del Paisaje. Los autores desarrollan tres dimesiones espaciales complementarias: espacial-formal, simbólica-ritual y social. Sus resultados apuntan al uso de una misma tradición técnica para su ejecución, la que fue eventualmente compartida por distintas generaciones entre los siglos $\vee$ y XIV. 
Ana Igareta, Guillermo López y Cecilia Morgan abordan el análisis de una pieza perteneciente a la colección del Museo de La Plata. Se trata de un vaso serpentiforme hallado en 1922 en el sitio Famabalasto, Catamarca, y que los autores consignan como parte de un ajuar funerario. La investigación les permitió proponer una interpretación alternativa a la caracterización previa de la iconografía de este vaso (como un ser fantástico de dos cabezas) y establecer que se trataría de dos individuos de una especie en particular, representados en un momento específico de su vida.

Siguiendo con las colecciones del Museo de La Plata, Helena Horta, Verónica Lema, María Teresa Plaza y Carolina Odone presentan el análisis de un único objeto: una tableta metálica peculiar debido a su iconografía y su confección. Las autoras proponen que, si bien la tableta es de manufactura colonial temprana, su iconografía remite a momentos prehispánicos, aunque pone en diálogo elementos andinos y europeos. En cuanto a su función, Horta et al. señalan que habría servido para la inhalación de sustancias psicoactivas y que podría haber sido utilizada en ritos relacionados con la fertilidad vegetal.

Cierra este segundo grupo, el artículo de Sheila Ali, Maricel Pérez, Patricia Bozzano y Silvia Domínguez, que presenta el análisis de mezclas de pigmentos usadas para la alfarería por los cazadores recolectores de la cuenca del río Paraná durante el Holoceno Tardío. Las autoras utilizan las técnicas MEB-EDX con el fin de identificar las materias primas colorantes de la fracción inorgánica de las muestras y contribuir a la discusión sobre la tecnología, contextos de uso y producción del color, relevando la presencia de hematita, fósforo, calcio, hidroxiapatita, hierro, manganeso y carbón vegetal.

El tercer grupo de artículos comienza con la contribución de Freddy Viñales, Claudia Ogalde, Juan Pablo Ogaldey Bernardo Arriaza, quienes estudian el rol social que tuvieron los aríbalos durante el Tawantinsuyu en el norte chileno. Los autores proponen una metodología que, por una parte, "divide" el soporte en tres secciones: cuello, cuerpo y base, para analizarlo, y por otra, identifica la iconografía para referirse finalmente a los hallazgos de restos vegetales contenidos en estas vasijas. Viñales et al. concluyen que los aríbalos sirvieron como negociadores entre los elementos culturales locales y los foráneos, dando cuenta de la identidad multiétnica del área.

Gonzalo Pimentel y Alonso Barros analizan los geoglifos y petroglifos que se encuentran en el sendero entre Tamentica y Quebrada de Los Pintados (Guatacondo, Tarapacá), con el propósito de indagar cómo los pueblos andinos prehispánicos inscribían su política de la memoria en los senderos. Reconociendo el influjo europeo, los autores determinan las diversas estrategias empleadas por los pueblos andinos para activar la geografía de la memoria y las huacas. Así, en el imaginario andino, Los Pintados y Tamentica fueron probablemente considerados importantes lugares sagrados o huacas, espacios que dan cuenta de una profunda ontología. En San Pedro Estación, Región de Antofagasta, Carolina Odone estudia la travesía de las 
cajas de madera de San Antonio de Padua. La autora las visualiza, por un lado, como agentes que actualizan distintas temporalidades históricas y sociales $y$, por otro, como objetos que sintetizaron colonialmente la vida de los indígenas en el contexto evangelizador. A pesar de ser un soporte originariamente europeo, las cajas de madera habrían entrado en la intimidad del espacio doméstico a partir de las campañas de extirpación de idolatrías, confiriéndole a estos objetos una gran capacidad de amalgamar vidas sociales.

En el último artículo de este número, Roberto Campbell, Francisca Moya y Renata Gutiérrez presentan los resultados del estudio de 26 sitios de arte rupestre en el sur chileno, constituyendo un corpus con motivos no figurativos de una data de 2000 años, mucho más abundante y variable -técnica y espacialmente- de lo que la literatura especializada ha reconocido. Los autores proponen la hipótesis de que esta variabilidad se debería a la inexistencia de un lenguaje visual compartido, al menos en lo que a arte rupestre se refiere, llegando a considerar que no es posible establecer un estilo general o local para este conjunto de sitios.

Cierra este número una contribución especial, pues se trata de un estudio que condensa las investigaciones de varias décadas, realizadas por una destacada antropóloga chilena: Verónica Cereceda. La autora aborda el problema de las capacochas, rituales de sacrificio que se realizaron en algunas de las cumbres andinas durante la época incaica y colonial. Cereceda concentra la mirada en un solo aspecto: el diseño tejido en las minúsculas prendas femeninas que forman parte de las ofrendas mortuorias. La autora nos lleva, a lo largo de un extenso y profundo recorrido, por el mundo del diseño textil andino, revelando las complejas tramas de la significación que se vuelven visibles gracias a la elección de colores, diseños y texturas, las que manifiestan, a su vez, diversas visiones de mundo y significados de intensa densidad.

Paula Martínez Sagredo*

* Investigadora Asociada, Universidad de Tarapacá, Departamento de Ciencias Históricas y Geográficas, Proyecto Fondecyt Iniciación 11190356. 